\title{
Nodular fasciitis: USP6-associated neoplasia through multiple pathways
}

\author{
Kazumi Fujioka \\ Department of Radiology, Nihon University School of Medicine, 30-1 Ohyaguchi-kamicho, Itabashi-ku, Tokyo 173-8610, Japan.
}

*Corresponding Author: Kazumi Fujioka, MD. PhD. Department of Radiology, Nihon University School of Medicine, 30-1 Ohyaguchikamicho, Itabashi-ku, Tokyo 173-8610, Japan.

Received Date: October 09, 2021; Accepted Date: October 25, 2021; Published Date: October 29, 2021

Citation: Kazumi Fujioka. (2021). Nodular fasciitis: USP6-associated neoplasia through multiple pathways. J Cancer Research and Cellular Therapeutics. 5(5); Doi: 10.31579/2640-1053/100

Copyright: ( 2021 Kazumi Fujioka, This is an open-access article distributed under the terms of the Creative Commons Attribution License, which permits unrestricted use, distribution, and reproduction in any medium, provided the original author and source are credited.

\begin{abstract}
Even though nodular fasciitis (NF) is benign and self-limited nature, the presentations of clinical, ultrasonographic, and pathological features have been described as mimicking sarcoma. Erickson-Johnson et al. suggested that ubiquitin-specific protease 6 (USP6) transcriptional upregulation may be the driving force behind the high proliferative activity and growth of NF. When the lesion showed the proliferative findings of the margin on both ultrasonography (US) and pathology, accompanied by clinically rapid growth, self-limited and/or regress course, NF could be strongly suggested as previously described. In this article, the author reviews the current knowledge of NF as USP6-associated neoplasia and also describes the therapeutic strategy in NF. In addition to the presentations of clinical, ulrtrasonographic, and pathological appearances of NF, the evaluation of percentage of USP6 break-apart FISH signals reflecting lifetime and mitotic counts in NF may be a potential procedure for accurate diagnosis in particularly young NF. It is putative that the inhibition of USP6-related genes might be the potential therapeutic strategies for the extremely rare malignant nodular fasciitis.
\end{abstract}

Keywords: nodular fasciitis; self-limited nature; USP6-associated neoplasia; high-resolution ultrasonography; USP6- related genes

\section{Introduction}

Erickson-Johnson et al. [1] suggested that USP6 transcriptional upregulation may be the driving force behind the high proliferative activity and growth and the consistent involutional nature of NF. The author previously reported the case series of NF and strongly suggested that proliferative findings of the margin of the nodule on US and pathological features are caused by the driving force of USP6 transcriptional upregulation behind the high proliferative activity and growth of NF from an established cytogenetic nature. In this article, the current knowledge of NF as USP6-related neoplasia will be reviewed. The author also describes the therapeutic strategy in USP6-associated neoplasia such as aneurysmal bone cyst (ABC) and NF.

\section{Transient neoplastic nature in nodular fasciitis}

$\mathrm{NF}$ is a benign soft tissue tumor of fibroblastic/myofibroblastic differentiation that was first described in 1955 by Konwaler et al. [2]. While, Goodlad et al. [3] documented the first case of dermal nodular fasciitis (DNF) in 1990. The author previously has reported a common subcutaneous NF [4] and a rare intradermal type [5]. Though this entity of the intradermal type have very rarely occurred, De Feraudy et al. [6] have studied intradermal nodular fasciitis as a rare lesion analyzed in a series of 24 cases, and recently the lesion involved both the dermis and subcutis as a superficial nodular fasciitis [7] and a dermally located lesion [8] have been reported in the dermatologic field. NF presents most typically in the upper extremities, the trunk, and the head and neck. It usually presents between the ages of 20 and 40 years, often with tenderness [4]. The subcutaneous, fascial, intramuscular, and rarely intradermal type have been found [5]. NF shows a peculiar clinical behavior characterized by rapid, self-limited growth and spontaneous regression after a few weeks. Pathologically, NF typically featured uniform spindled cells arranged in irregularity intersecting short fascicles, and occasional storiform patterns. The cells contained plump, spindled to stellate nuclei with fine to open chromatin and small nucleoli. The background stroma ranged from myxoid to collagenous [4]. The characteristic tissue culture-like, feathery myxoid appearance was present at least focally [9]. Immunohistochemically, spindle cells in NF contain vimentin, muscle-specific action, and smooth muscle-specific actin [10], whereas it is known that NF is negative for several markers such as desmin, keratin, or S-100 protein in contrast to sarcoma [11]. Due to the aggressive clinical presentation, malignant ultrasonograpic appearances, and the histopathology with its high cellularity and high mitotic activity, NF can be misdiagnosed as a malignant soft tissue tumor, often leading to 
unnecessarily aggressive therapy [12].

\section{High-resolution ultrasonography appearances in nodular fasciitis}

Recently, dermatologic ultrasound imaging has been growing because of the development of high-resolution multifrequency transducers and multichannel color Doppler machines [13]. In dermatology, a $15 \mathrm{MHz}$ is the minimum frequency recommended for performing dermatologic examination by the international working group or DERMUS (Dermatologic Ultrasound) [13, 14]. The author has reported a subcutaneous NF representing compressed fascia using both highresolution ultrasonography (US) and magnetic resonance imaging (MRI) appearances [4] and a rare case of intradermal type showing the primarily in the dermis accompanied by the dilatation and proliferation of the vessels using high-resolution color Doppler ultrasound [5], it is known that high- resolution US can clearly represent the location of the dermis and subcutaneous, association of the fascia, and the presence of dilation and proliferation of the vessels. The author usually performs US studies for dermatologic fields with a high-resolution, broad-band $(5 \mathrm{MHz}-$ $18 \mathrm{MHz}$ ) linear transducer and described several studies [4, 5, 15-29].

\section{Presentations of clinical, ultrasonographic, and pathological features of nodular fasciitis from an established cytogenetic viewpoint}

Even though NF is benign and self-limited, the clinical, ultrasonographic and pathological presentations have been described as mimicking sarcoma. Erickson-Johnson et al. [1] suggested that USP6 transcriptional upregulation may be the driving force behind the high proliferative activity and growth of NF. The author previously reported that irregular or lobular margin on US and poorly defined margin on histology is attributed to the cytogenetic nature of NF [15]. Furthermore, the research results strongly suggested that proliferative findings the margin of the nodule on US and pathological features are caused by the driving force of USP6 transcriptional upregulation behind the high proliferative activity and growth of NF from an established cytogenetic nature [17]. When the lesion showed the proliferative findings of the margin on both US and pathology, accompanied with clinically rapid growth, self-limited and/or regress course, NF could be strongly suggested as previously described [17].

\section{Fusion patterns of USP6 in nodular fasciitis}

A clear relationship between NF and a recurrent genetic abnormality had been established in FISH analysis [1, 9, 30-33]. Genomic rearrangements of the USP6 locus were found in 92\% of NF. Rapid amplification of 5'cDNA ends identified MYH9 as the transcription partner. The fusion of the MYH9 promoter region to the entire coding region of USP6 was shown using RT-PCR and direct sequencing [1]. The result indicated that the MYH9-USP6 fusion was demonstrated in 12 of 16 (75\%) [1]. USP6 oncogenes was also detected in aneurysmal bone cyst $(\mathrm{ABC})[34,35]$, suggesting that these histological manifestations in $\mathrm{ABC}$ and $\mathrm{NF}$ may have a common pathogenic denominator mediated by USP6 transcription upregulation [1]. Regarding $\mathrm{ABC}$, Ye et al. reported the USP6-induced expression of matrix metalloproteases through activation of $\mathrm{NF}-\kappa \mathrm{B}$, a transcription factor with a key role in inflammation and proliferation [36]. Oliveira et al. suggested that $\mathrm{ABC}$ and $\mathrm{NF}$ reside in the same biologic spectrum, namely USP6-induced tumors [37]. Shin et al. reported that FISH analysis for USP6 had a sensitivity of $86 \%$ and specificity of $100 \%$ for a diagnosis of NF [9]. While, Erber et al. reported that the sensitivity and specificity of USP6 FISH for NF was $74.4 \%$ and $100 \%$, respectively [31]. These studies indicate a clear association between NF and a recurrent genetic abnormalities in FISH analysis.

MYH9 is located on chromosome 22q12.3-q13 [1] and it is the most frequent fusion partner [1, 33]. RRBP1, CALU, CTNNB1, MIR22HG,
SPARC, THBS2, and COL6A2 as less frequent gene partners has been detected [32]. These studies demonstrated the neoplastic nature of NF and USP6-associated neoplasia [32]. SEC31A, EIF5A, COL1A1, COL1A2, PAFAH1B1, SERP1NH1, COL3A1 have been also identified [38-45]. Regarding EIF5A, the USP6 gene rearrangement was shown by using FISH and a subsequent analysis demonstrated a novel EIF5A-USP6 gene fusion by means of the Archer fusionPlex Sarcoma kit [8]. They mentioned that the genesis of NF is also caused by Wnt signaling pathway which is the essential target of USP6 [8]. PPP6R3-USP6 fusion has been shown in two cases of NF accompanied with malignant manifestation [44, 45].Regarding the cutaneous NF, Kumar et al. [30] have described the presence of the USP6 rearrangement and MYH9-USP6 fusion, suggesting that the similar cytogenetic nature has been revealed both in subcutaneous NF and intradermal type.

\section{Nodular fasciitis as USP6-associated neoplasia through pathway}

Due to the identification of structural similar USP6 fusion gene in both $\mathrm{ABC}$ and $\mathrm{NF}$, it is suggested that these diseases are clonal neoplasia that may have the same biological spectrum [37]. Myositis ossificans (MO) and fibro-osseous pseudotumor of digits (FOPD) also show the identification of USP6 gene fusion, indicating that these lesions may present the same entity. It is known that multiple fusion patterns of USP6 lead to transcriptional activation of USP6 [46, 47].

Regarding the signaling pathways, USP6 induces several signal pathways including $\mathrm{NF} \kappa \mathrm{B}, \mathrm{Wnt} / \beta$-catenin, and JAK-1-STAT3 during tumorigenesis $[46,48,49]$. The important role of Jak1-STAT3 signaling in USP-6-related tumorigenesis has been reported [48], whereas Madan et al. [49] described that USP6 oncogene promotes Wnt signaling by deubiquitylating Frizzleds. Recently, Li et al. [50] reported that c-Jun is a novel substrate of USP6 and might serves as an essential role of NF.

Regarding Wnt signaling pathway [49], it has been noted that chromosomal translocations of NF cause USP6 overexpression, that in turn lead to transcriptional activation of the $\mathrm{Wnt} / \beta$-catenin pathway. Inhibition of Wnt signaling pathway significantly attenuated the growth of USP6-driven xenograft tumors, suggesting that Wnt signaling is an essential target of USP6 in tumorigenesis of NF. The study by Madan et al. [49] revealed a new mechanism for pathological Wnt pathway activation in NF and $\mathrm{ABC}$ and suggested a novel treatment method to modulate Wnt signaling. With respect to the Jak1-STAT3 signal, Quick et al. [48] showed that the Jak1-STAT3 signals are potential effectors of the USP6-TRE17 oncogene in the tumorigenesis of ABC and NF. They showed that USP6 activates Jak1-STAT3 pathway by deubiquitinating Jak-1, resulting in the stabilization and STAT3 activation. It has been suggested that the tumorigenic activity of USP6 was decreased by a JAK family inhibitor, or by CRISPR (Clustered Regularly Interspaced Short Palindromic Repeats)-mediated deletion of JAK1 or STAT3 [48]. It has been suggested that studies demonstrating the downstream effects of USP6 may contribute to the therapeutic strategy in patients with $\mathrm{ABC}$ and NF [50]. The novel genes, namely the Frizzled and JAK1 genes were recently demonstrated as USP6 substrates [48, 49]. While, Li et al. [50] described the transcription factor c-Jun as a novel substrate of USP6. It has been reported that USP6 stabilized the c-Jun protein and potentiated c-Jun-dependent downstream signaling and cell invasion. They suggested that the interaction between USP6 and c-Jun might be important in patients with NF. It is known that USP6 has been shown to have potential roles in $\mathrm{ABC}$ and NF. Therefore they also suggest that the inhibition of $\mathrm{c}-$ Jun and other USP6-associated genes including JAK1 and Frizzled genes would produce the benefit in the patients with $\mathrm{ABC}$ and NF. The author suggests that the inhibition of c-Jun and other USP-related genes may be the potential therapeutic strategies for recurrent and/or inoperable ABC. Whereas the most of NF is benign, self-limited process including high proliferative growth activity and regress nature. Extremely rare malignant 
behavior of NF [44, 45] shown by USP6-PPP6R3 gene fusion has been reported. Based on the evidence, the author also suggests that the inhibition of USP6-related genes might be essential therapeutics in malignant behavior type of NF.

\section{Self-limited nature of NF from USP6-FISH (\%) viewpoint}

The report [51] indicated that young NF defined as preoperative duration $<1$ month contains a high percentage of USP6 break-apart FISH signals than old NF defined as preoperative duration $>3$ months, suggesting that the result may demonstrate the connection with the self-limited nature of NF. The result indicated that in old NF, the percentage of USP6 breakapart FISH signals can be as low as $14-27 \%$. It is putative that USP6 break-apart FISH signals reflect the growth activity and regress nature in NF. In addition to the presentations of clinical, ulrtrasonographic, and pathological appearances, the evaluation of percentage of USP6 breakapart FISH signals may be a potential procedure for diagnosis in young NF $[17,51]$. In old NF, the author suggests that accurate diagnosis should be comprehensively made by the presentation of clinical, ultra sonographic, pathological, and cytogenetic features. The result showed a strong inverse relationship between the lifetime and percentage of USP6 break-apart signals and a strong positive association between USP6 break-apart signals and mitotic counts [51]. Based on the evidence, the author suggests that NF has the self-limited behavior so-called the proliferative activity and regression nature. It has been known that USP6 activation by promoter-swapping is characteristic feature accompanied with involvement of JAK-1/STAT1/STAT3 pathways leading to stabilization of the JUN protein [51]. In their research, the mRNA level of USP6, TRAIL, IFN-beta, JAK1, STAT1, STAT3, JUN, and CDKN2A as the pathways of the tumorigenesis of NF was evaluated using qRTPCR, resulting in the significantly negative correlations between USP6FISH and JAK1 and between USP6-FISH and STAT1. They also described that the role of the JAK-1/STAT1/STAT3 pathway is "active" during the whole lifetime of NF. While, it has been also suggested that cellular senescence through CDKN2A/p16 could be responsible for selflimited nature and a negative feedback mechanism that USP6 induces TRAIL-mediated apoptosis was revealed $[52,53]$. They described that in young NF, the nuclear localization of TRAIL was shown and in old type, the dominant membraneous-cytoplasmic TRAIL positivity was represented [51].

\section{Conclusion}

In addition to the presentation of clinical, ulrtrasonographic, and pathological appearances, the evaluation of percentage of USP6 breakapart FISH signals reflecting lifetime and mitotic counts in nodular fasciitis may be a potential procedure for accurate diagnosis in particularly young nodular fasciitis.

It is putative that the inhibition of USP6-related genes might be the potential therapeutic strategies for recurrent and/or inoperable aneurysmal bone cyst and extremely rare malignant nodular fasciitis.

\section{References}

1. Erickson-Johnson MR, Chou MM, Evers BR, Roth CW, Seys AR, Jin L, et al. (2011). Nodular fasciitis: a novel model of transient neoplasia induced by MYH9-USP6 gene fusion. Lab Invest. 91:1427-1433.

2. Konwaler BE, Keasbey L, Kaplan L. (1955). Subcutaneous pseudosarcomatous fibromatosis (fasciitis). Am J Clin Pathol. 25:241-252.

3. Goodlad JR, Fletcher CDM. (1990). Intradermal variant of nodular 'fasciitis'. Histopathology. 17:569-571.
4. Fujioka K, Fujioka A, Eto H, Suzuki K, Sanuki E, Tanaka Y. (2006). Nodular fascitis in the thigh followed up using ultrasonography. J Med Ultrason. 33:49-53.

5. Fujioka K, Fujioka A, Oishi M, Eto H, Tajima S, Nakayama T. (2017). Ultrasonography findings of intradermal nodular fasciitis; a rare case report and review of the literature. Clin Exp Dermatol. 42:335-336.

6. De Feraudy S, Fletcher CD. (2010). Intardermal nodular fasciitis: a rare lesion analyzed in a series of 24 cases. Am J Surg Pathol. 34: 1377-1381.

7. Maloney N, Leblanc RE, Sriharan A, Bridge JA, Linos K (2019). Superficial nodular fasciitis with atypical presentations: report of 3 cases and review of recent molecular genetics. Am J Dermatopahtol. 41: 931-936.

8. Lenz J, Michal M, Svajdler M, Ptakova N, Lenz D, Konecna P, et al. (2020). Novel EIF5A-USP6 gene fusion in nodular fasciitis associated with unusual pathologic features: a report of a case and review of the literature. Am J Dermatopathol. 42: 539-543.

9. Shin C, Low I, Ng D, Oei P, Miles C, et al. (2016). USP6 gene rearrangement in nodular fasciitis and histological mimics. Histopathology. 69: 784-791.

10. Borumandi F, Cascarini L, Mallawaarachchi R, Sandison A. (2012). The chameleon in the neck: nodular fasciitis mimicking malignant neck mass of unknown primary. Int J Surg Case Rep. 3: 501-503.

11. Lloyd AA, Witheiler, Menter A. (2015). Nodular fasciitis of the lip mucosa: a rare but clinically important entity. Clin Exp Dermatol. 40:408-412.

12. Nishio J. (2013). Updates on the cytogenetics and molecular cytogenetics of benign and intermediate soft tissue tumors. Oncol Lett. 5: 12-18.

13. Wortsman $\mathrm{X}$, Alfageme $\mathrm{F}$, Roustan $\mathrm{G}$, Arias-Santiago $\mathrm{S}$, Martorell A, et al. (2016). Guidelines for performing dermatologic ultrasound examinations by the DERMUS group. J Ultrasound Med. 35:577-580.

14. Wortsman X, Alfageme F, Roustan G, Arias-Santiago S, Martorell A, et al. (2016). Proposal for an assessment training program in dermatologic ultrasound by the DERMUS group. $\mathbf{J}$ Ultrasound Med. 35:2305-2309.

15. Fujioka K, Fujioka A, Tajima S, Oishi M, Hayashi K, Eto H, et al. (2018). Characteristic power Doppler sonographic imaging of nodular fasciitis from dermatological perspective: another case and review of three cases. J Clin Case Rep. 8:1165.

16. Fujioka K, Fujioka A, Oishi M, Hayashi K, Nakayama T. (2018). High-resolution ultrasound imaging for angioleiomyoma: a painful and vascularized superficial tumor. Biomed J Sci \& Tec Res 9(5).

17. Fujioka K. (2018) Presentations of clinical, ultrasonographic and pathological features of nodular fasciitis from an established cytogenetic viewpoints: review of the case series. J Carcinog Mutagen 9: 326.

18. Fujioka K. (2018). Usefulness of comprehensive high-resolution ultrasound imaging in dermatologic field: epidermal cyst. Biomed J Sci \& Tec Res 12(1) .26717/BJSTR.2018.11.002184.

19. Fujioka K. (2019). Painful cutaneous and subcutaneous tumors accompanied with vascularized appearance using high-resolution ultrasound in dermatology: the acronym "ENGLAND" or "LEND AN EGG". Biomed J Sci \& Tec Res 12(4) /BJSTR.2019.12.002297.

20. Fujioka K. (2019). Real- time Tissue elastography in epidermal cyst: along with enhanced MRI and elasticity features of peripheral rim. Biomed J Sci \& Tec Res 16(5) .2019. BJSTR. MS.ID.002904. 
21. Fujioka K. (2019). A comparison between superficial and deepseated lipomas on high-resolution ultrasonography: with RTE and MRI appearances. Biomed J Sci \& Tec Res 19(2) .2019. BJSTR. MS.ID.003278.

22. Fujioka K. (2019). Characteristic appearances of nodular fasciitis on high-resolution ultrasonography: with vasculature status from a lesion-located perspective. Biomed J Sci \& Tec Res 20(4) .2019. BJSTR. MS.ID.003490.

23. Fujioka K. Fujioka A, Oishi M, Okada M. (2019). A new application in dermatological ultrasound. Biomed J Sci \& Tec Res 22(5) .2019. BJSTR. MS.ID.003809.

24. Fujioka K. Fujioka A, Okada M. (2019). Utility of high-resolution ultrasonography by using acoustic coupler in dermatology. Biomed J Sci \& Tec Res 23(5) .2019. BJSTR. MS.ID.003953.

25. Fujioka K. Fujioka A, Okada M. (2020). High-resolution ultrasonographic appearances in superficial fibromatoses: palmar and plantar diseases. Biomed J Sci \& Tec Res 27(2)-2020. BJSTR. MS. ID. 004462.

26. Fujioka K. (2020). Relationship between high-resolution ultrasonographic and pathological appearances in epidermal cyst: an image representation of the plantar type. Journal of Clinical Case Reports Online 1: (1) 1004.

27. Fujioka K. (2020). Subtypes of subcutaneous angioleiomyoma on high-resolution color Doppler ultrasonography along with MRI appearances. Biomed J Sci \& Tec Res 31(1).

28. Fujioka K. (2020). Characteristic appearances of peripheral schwannoma on high-resolution ultrasonography and MRI: comparison between Antoni A-dominant and Antoni B-dominant types. Biomed J Sci \& Tec Res 32(2) .2020. BJSTR. MS.ID.005221.

29. Fujioka K. (2021). Acral calcified angioleiomyoma along with radiological features. Biomed J Sci \& Tec Res 38(2) .2021. BJSTR. MS.ID.006112.

30. Kumar E, Patel NR, Demicco EG, Bovee JVMG, Olivera AM, Lopez-Terrada DH, et al. (2016). Cutaneous nodular fasciitis with genetic analysis: a case series. J Cutan Pathol. 43: 1143-1149.

31. Erber R, Agaimy A. (2018). Misses and near misses in diagnosing nodular fasciitis and morphologically related reactive myofibroblastic proliferations: experience of a referral center with emphasis on frequency of USP6 gene rearrangements. Virchows Arch. 473: 351-360.

32. Patel NR, Chrisinger JSA, Demicco EG, Sarabia SF, Reuther J, Kumar E, et al. (2017). USP6 activation in nodular fasciitis by promoter-swapping gene fusions. Mod Pathol. 30: 1577-1588.

33. Amary MF, Ye H, Berisha F, Tirabosco R, Presneau N, et al. (2013). Detection of USP6 gene rearrangement in nodular fasciitis: an important diagnostic tool. Virchows Arch. 463: 9798.

34. Oliveira AM, Perez-Atayde AR, Inwards CY, Medeiros F, Derr V, et al. (2004). USP6 and CDH11 oncogenes identify the neoplastic cell in primary aneurysmal bone cysts and are absent in so - called secondary aneurysmal bone cysts. Am J Pathol. 165: 1773-1780.

35. Oliveira AM, Perez-Atayde AR, dal Cin P, Gebhard MC, Chen CJ, et al. (2005). Aneurysmal bone cyst variant translocations upregulate USP6 transcription by promoter swapping with the ZNF9, COL1A1, TRAP150, and OMD genes. Oncogene. 24: 3419-3426.

36. Ye Y, Pringle LM, Lau AW, et al. (2010). TRE17/USP6 oncogene translocated in aneurysmal bone cyst induces matrix metalloproteinase production via activation of NF-kappaB. Oncogene. 29:3619-3629.
37. Oliveira AM, Chou MM. (2014). USP6-induced neoplasms: the biologic spectrum of aneurysmal bone cyst and nodular fasciitis. Human Pathology. 45: 1-11.

38. Lam SW, Cleton-Jansen AM, Cleven AHG, Ruano D, van Wezel T, Szuhai K, et al. (2018). Molecular analysis of gene fusions in bone and soft tissue tumors by anchored multiplex PCR-based targeted next-generation sequencing. J Mol Diagn. 20: 653-663.

39. Wang JC, Li WS, Kao YC, Lee JC, Lee PH, Huang SC, et al. (2021). Clinicopathological and molecular characterisation of USP6-rearranged soft tissue neoplasms: the evidence of genetic relatedness indicates an expanding family with variable boneforming capacity. Histopathology. 78:676-689.

40. Qiu Y, Peng R, Chen H, Zhuang H, He X, Zhang H, et al. (2021). Atypical nodular fasciitis with a novel PAFAH1B1-USP6 fusion in a 22-month -old boy. Virchows Arch. 479: 623-629.

41. Nakayama $S$, Nishio J, Aoki M, Koga K, Nabeshima K, Yamamoto T. (2021). Ubiquitin-specific peptidase 6 (USP6)associated fibroblastic/myofibroblastic tumors: evolving concepts. Cancer Genomics \& Proteomics. 18: 93-101.

42. Lu Y, He X, Qiu Y, Chen H, Zhuang H, Yao J, et al. (2020). Novel CTNNB1-USP6 fusion in intravascular fasciitis of the large vein identified by next-generation sequencing. Virchows Arch. 477: 455-459.

43. Paulson VA, Stojanov IA, Wasman JK, Restrepo T, Cano S, Plunkitt J, et al. (2020). Recurrent and novel USP6 fusions in cranial fasciitis identified by targeted RNA sequencing. Mod Pathol. 33: 775-780.

44. Guo R, Wang X, Chou MM, Asmann Y, Wenger DE, Allbraheemi A, Molavi DW, et al. (2016). PPP6R3-USP6 amplication: novel oncogenic mechanism in malignant nodular fasciitis. Genes Chromosomes Cancer. 55: 640- 649.

45. Teramura Y, Yamazaki Y, Tanaka M, Sugiura Y, Takazawa Y, Takeuchi K, et al. (2019). Case of mesenchymal tumor with the PPP6R3-USP6 fusion, possible nodular fasciitis with malignant transformation. Pathol Int. 69: 706-709.

46. Pringle LM, Young R, Quick L, Riqueime DN, Oliveria AM, May MJ, Chou MM. (2012). Atypical mechanism of NF- $\kappa$ B activation TRE17/ubiquitin -specific protease 6 (USP6) oncogenes and its requirements in tumorigenesis. Oncogene. 31: 3525-3535.

47. Hiemcke-Jiwa, LS, van Gorp JM, Fisher C, Creytens D, van Diest PJ, Flucke U. (2020). USP-associated neoplasms: a rapidly expanding family of lesions. Int J Surg Pathol. 28: 816-825.

48. Quick L, Young R, Henrich IC, et al. (2016). Jak-1-STAT3 signals are essential effectors of the USP6/TRE17 oncogenes in tumorigenesis. Cancer Res. 76: 5337-5347.

49. Madan B, Walker MP, Young R, Quick L, Orgel KA, Ryan M, et al. (2016). USP6 oncogene promotes Wnt signaling by deubiquitylating Frizzleds. Proc Natl Acad Sci USA. 113: 29452954.

50. Li L, Yang H, He Y, Li T, Feng J, Chen W, et al. (2018). Ubiquitin-specific protease USP6 regulates the stability of the cJun protein. Mol Cell Biol. 38(2): e00320-00317.

51. Sápi Z, Lippai Z, Papp G, Hegyi L, Sápi J, Dezsó K, et al. (2021). Nodular fasciitis: a comprehensive, time-correlated investigation of 17 cases. Mod Pathol.

52. Matsuda I, Nakamura J, Ohkouchi M, Torii Y, Futani H, Tsukamoto Y, et al. (2019). Expression of p16 in nodular fasciitis: an implication for self-limited and inflammatory nature of the lesion. Int J Clin Exp Pathol. 12: 1029-1034.

53. Henrich IC, Young R, Quick L, Oliveira AM, Chou MM. (2018). USP6 confers sensitivity to IFN-mediated apoptosis through modulation of TRAIL signaling in Ewing sarcoma. Mol Cancer Res. 16: 1834-1843. 
This work is licensed under Creative

Commons Attribution 4.0 License

To Submit Your Article Click Here: Submit Manuscript

DOI: $10.31579 / 2640-1053 / 100$
Ready to submit your research? Choose Auctores and benefit from:

$>$ fast, convenient online submission

$>$ rigorous peer review by experienced research in your field

$>$ rapid publication on acceptance

$>$ authors retain copyrights

$>$ unique DOI for all articles

$>$ immediate, unrestricted online access

At Auctores, research is always in progress.

Learn more auctoresonline.org/journals/cancer-research-and-cellulartherapeutics 